\title{
APOE and COMT polymorphisms are complementary biomarkers of status, stability, and transitions in normal aging and early mild cognitive impairment
}

\author{
Roger A. Dixon 1,2*, Correne A. DeCarlo ${ }^{3}$, Stuart W. S. MacDonald ${ }^{3}$, David Vergote ${ }^{4}$, \\ Jack Jhamandas ${ }^{2,5}$ and David Westaway ${ }^{2,4}$ \\ ${ }^{1}$ Department of Psychology, University of Alberta, Edmonton, AB, Canada \\ ${ }^{2}$ Neuroscience and Mental Health Institute, University of Alberta, Edmonton, AB, Canada \\ ${ }^{3}$ Department of Psychology, University of Victoria, Victoria, BC, Canada \\ ${ }^{4}$ Centre for Prions and Protein Folding Diseases, University of Alberta, Edmonton, AB, Canada \\ ${ }^{5}$ Department of Medicine (Neurology), University of Alberta, Edmonton, AB, Canada
}

\section{Edited by:}

Isidro Ferrer, University of

Barcelona, Spain

Reviewed by:

Changiz Geula, Northwestern

University, USA

Katrin Beyer, Fundación Instituto de Investigación Biomédica Germans

Trias i Pujol, Spain

\section{*Correspondence:}

Roger A. Dixon, Department of

Psychology, P-217 Biological

Sciences Building, University of

Alberta, Edmonton, $A B$,

T6G 2E9, Canada

e-mail: rdixon@ualberta.ca
Objective: Research has reported associations among selected genetic susceptibility biomarkers and risk of (a) normal cognitive aging decrements, (b) established mild cognitive impairment (MCl), and (c) sporadic Alzheimer's disease (AD). In focusing on the transitional normal-to-early $\mathrm{MCl}$ phase, we examine associations among three theoretically relevant polymorphisms (APOE [rs429358, rs7412], BDNF [rs6265], COMT [rs4680]) and both baseline cognitive status ( $\mathrm{MCl}$ vs. normal aging) and two-wave (four-year) longitudinal stability or change profiles. The latter included three profiles: (a) stable as normal aging, (b) stable or chronic impairment (MCl-to-MCl), and (c) emergence of impairment (normal-to-MCl).

Method: Genotyped older adults ( $n=237$ at baseline; age range $=64-91 ; 62 \%$ women) from the Victoria Longitudinal Study were examined for (a) independent and interactive associations of three genetic polymorphisms with (b) two objectively classified cognitive status groups (not-impaired controls $(\mathrm{NIC})$ and $\mathrm{MCl}$ ) at (c) both baseline and across a two-wave (four-year) longitudinal interval.

Results: First, logistic regression revealed that the presence of at least one APOE $\varepsilon 4$ allele (the risk factor for $A D$ ) was linked to greater baseline risk of objective $\mathrm{MCl}$. Second, multinomial logistic regression revealed that (a) the presence of an APOE $\varepsilon 4$ allele was associated with an increased risk of 4-year $\mathrm{MCl}$ status stability (chronicity), and (b) the COMT homozygous risk genotype (G/G or Val/Nal) was associated with an increased risk of both $\mathrm{MCl}$-to-MCl stability (chronicity) and emerging $\mathrm{NIC}$-to-MCl conversion.

Discussion: Both chronicity and emergence of objectively classified early cognitive impairment may be genetically heterogeneous phenomena, with influences from a panel of both normal cognitive aging (COMT) and AD-related (APOE) polymorphisms.

Keywords: $A P O E, B D N F, C O M T$, mild cognitive impairment, Victoria Longitudinal Study

\section{INTRODUCTION}

The often lengthy and subtle pathophysiological changes linking normal aging and diagnosable sporadic (late-onset) Alzheimer's disease (AD) are continuous, differentiable, detectable, and worthy of study as a classifiable phase in growing numbers of older adults (e.g., Albert et al., 2011; McKhann et al., 2011; Sperling et al., 2013). Known as mild cognitive impairment (MCI), the key clinical characteristics, markers, and classification procedures have evolved toward a cluster of shared perspectives, related methods, and clinical health implications (e.g., Ritchie et al., 2001; Winblad et al., 2004; Petersen and Knopman, 2006; Albert et al., 2011; Geda and Nedelska, 2012; Lin and Neumann, 2013; Sperling et al., 2013). Briefly, summary principles include: (a) $\mathrm{MCI}$ is defined as a mild but clinically relevant neurocognitive dysfunction emerging in a dynamic and variable distribution between normal decline and dementia; (b) biomarkers and risk factors for MCI-related transitions can lead to preclinical targets for earlier interventions to prevent or delay $\mathrm{AD}$; and (c) much work is needed to classify, track, and identify markers of MCIrelated transitions (Payton, 2006; Golde et al., 2011; Abner et al., 2012; Hurd et al., 2013; Risacher et al., 2013).

Recent research has focused on detecting biological (e.g., genetic risk) and environmental (e.g., lifestyle) factors that discriminate cognitively normal older adults from MCI groups (e.g., Woodard et al., 2012; Anstey et al., 2013). New longitudinal data have revealed evidence of varying stabilities in status, especially in MCI transitions (e.g., Albert et al., 2011; Dolcos et al., 2012; Risacher et al., 2013). This implies that MCI status may 
be a dynamic, heterogeneous, multi-faceted, and variable phenomenon (Palmer et al., 2002; Koepsell and Monsell, 2012). By definition, MCI involves relatively lower levels of performance or accelerated decline-but not deficits that interfere with everyday functioning or that merit a dementia diagnosis (Albert et al., 2011).

Recommended longitudinal research requires several key classification elements. First, initial status assessment could be confirmed independently at a second session separated by a clinically meaningful interval (e.g., Vandermorris et al., 2011). At baseline, early or transitional MCI classifications may not be etiologically pure, diagnostically firm, or projected to follow an inevitable path to dementia. Second, the phenotypic heterogeneity and multidirectionality of normal cognitive aging (e.g., Dixon et al., 2012; Josefsson et al., 2012) may be reflected also in early transition phases of MCI (Koepsell and Monsell, 2012). Third, multiple factors (e.g., genetic, physiological, health, environmental) contribute to a wide range of normal and early-impaired cognitive aging changes (Fotuhi et al., 2009; Anstey, 2014). Fourth, markers may be independently or interactively (e.g., synergistically) used to discriminate normal aging and early MCI (e.g., Forlenza et al., 2010; Lanni et al., 2012; Risacher et al., 2013). Fifth, classifying individuals in provisional MCI status requires assessments that (a) differentiate normal aging decrements from those prodromal for dementia, (b) produce repeatable (stable) status over longitudinal time, (c) are sensitive to early transitions that emerge during the study period, or $(\mathrm{d})$ are associated with eventual conversion to $\mathrm{AD}$ or a related disorder. Finally, MCI classification schemes can be characterized by the extent to which they fulfill these validityrelated process and outcome criteria (e.g., Gauthier et al., 2006; Dolcos et al., 2012).

We used objective criteria to identify cognitive deficits vis-àvis age and education level (e.g., Ritchie et al., 2001; Dixon et al., 2007; de Frias et al., 2009; Plassman et al., 2010; Dolcos et al., 2012). These objective-based procedures coordinate with consensus recommendations but are also transferable across research settings, including clinics, labs, and data archives. For example, using these objective classification procedures we found that select functional biomarkers (e.g., blood pressure, body mass index) could (a) differentiate not-impaired control (NIC) from MCI groups at baseline, (b) establish high levels (70-80\%) of status and cognitive stability over a 4 -year longitudinal period, (c) provide sensitivity to subtle status transitions (de Frias et al., 2009; Dolcos et al., 2012; Dixon and de Frias, 2014), and (d) relate to underlying neurobiological markers via metabolomics analyses (Zheng et al., 2012). Informing present research on genetic markers of non-demented aging and MCI are studies of both susceptibility genes for sporadic (late-onset) AD (e.g., Bertram et al., 2007) and candidate genetic associations and interactions (e.g., Martinez et al., 2009; Harris and Deary, 2011; Laukka et al., 2013; Sapkota et al., 2014). Examining associations with emerging MCI is a promising research direction (Albert et al., 2011; Brainerd et al., 2011; Izaks et al., 2011; Risacher et al., 2013; Caselli et al., 2014). We now test the role of specific genetic markers of normal cognitive aging and $\mathrm{AD}$, as they relate to early phases of mild cognitive impairment status and change, with the general goal of promoting early detection with its promising intervention possibilities (Reinvang et al., 2010; Risacher et al., 2013).

Both non-demented cognitive aging and sporadic $\mathrm{AD}$ are genetically heterogeneous (Harris and Deary, 2011). Early MCI conditions may reflect genetic influences from polymorphisms related to both normal cognitive changes and $\mathrm{AD}$ outcomes. We assembled new data from the Victoria Longitudinal Study (VLS) to test whether three commonly associated genetic markers of normal cognitive aging or neurocognitive dysfunction are also associated with objectively classified (a) early MCI status (vs. non-demented aging) at baseline, (b) a 4-year MCI-related status stability profile reflecting chronicity of impaired status, and (c) a 4-year MCI-related status profile reflecting a transitional phase from normal aging to MCI. Three genes commonly implicated in aging and neurocognitive functioning are Apolipoprotein E (APOE, rs429358 and rs7412), Brain-derived Neurotrophic Factor (BDNF, rs6265), and Catechol-O-Methyl Transferase (COMT, rs4680). APOE is involved in lipoprotein metabolism. The well-known $\varepsilon 4$ variant is the largest known genetic risk factor of sporadic $\mathrm{AD}$ and has been implicated in normal cognitive aging decline (e.g., Jorm et al., 2007; Kozauer et al., 2008; Wisdom et al., 2011; Schiepers et al., 2012; Davies et al., 2014). The other two alleles of APOE are $\varepsilon 2$ and $\varepsilon 3$, with (a) the former considered potentially protective and (b) the latter considered neutral for cognitive and neurodegenerative disease. The molecular foundations of BDNF and COMT associations with neurocognitive performance have been well-described (e.g., Savitz et al., 2006). Briefly, COMT codes for an enzyme that degrades catecholamine neurotransmitters such as dopamine in the synaptic cleft. The polymorphism is represented by a valine (Val or G) and methionine (Met or A) substitution, with the latter reducing dopamine degradation. COMT Val carriers (both Val-Val and Val-Met) are considered relatively at risk for specific cognitive deficits with aging (e.g., Barnett et al., 2008; Wishart et al., 2011) and possibly MCI (Martinez et al., 2009; Lanni et al., 2012). BDNF is a neurotrophic factor involved in neuronal survival and plasticity. BDNF expression declines with aging and it is associated with memory performance. The single polymorphism is represented by a Val and Met substitution, with the latter associated with lower hippocampal volume and reduced memory (and cognitive) performance in aging (Miyajima et al., 2008; Tapia-Arancibia et al., 2008), although direct associations are inconsistent (Forlenza et al., 2010; Mandelman and Grigorenko, 2012). For BDNF, Met carriers (both $\mathrm{A} / \mathrm{A}$ and $\mathrm{A} / \mathrm{G}$ ) are at risk. In this study, each of the genes offered potential risk for MCI status classification and MCI status stability over time [i.e., $A P O E$ risk $=\varepsilon 4$ carrier; COMT risk $=$ Val carrier $(\mathrm{G} / \mathrm{G}$ and $\mathrm{A} / \mathrm{G}) ; B D N F$ risk $=$ Met carrier $(\mathrm{A} / \mathrm{A}$ and $\mathrm{A} / \mathrm{G})]$.

Of the three genetic polymorphisms, APOE is the theoretically most likely associate of MCI status and stability (Brainerd et al., 2011; Harris and Deary, 2011). Several recent studies that linked $A P O E \& 4$ status to relatively late risk of preclinical progression to $\mathrm{AD}$ (e.g., Lane et al., 2008; Barabash et al., 2009; Elias-Sonnenschein et al., 2011; Reinvang et al., 2013). Until recently the $\varepsilon 4$ variant (including $\varepsilon 4 / \varepsilon 4$ and $\varepsilon 4 / \varepsilon 3$ combinations) has been inconsistently linked to MCI status, likely reflecting both clinical and methodological differences in status classification 
(e.g., Brainerd et al., 2011; Risacher et al., 2013). The present study extends a growing collection of results in several ways. First, complementing other studies (e.g., Brainerd et al., 2011; Anstey et al., 2013), we focus on older adults enrolled in a longitudinal study (ages 65-91 at baseline). Second, we examine concurrent and two-wave cognitive status stability relationships. Third, we used a standard and fully objective procedure for MCI classification, which may be useful for detecting early preclinical manifestations of impairment and potentially informative for translational goals. Fourth, in addition to $A P O E$, we include two selected genetic polymorphisms, both of which are prominently linked with cognitive phenotypes in normal aging (but rarely with MCI) (Harris and Deary, 2011). However, both BDNF and COMT have been reported as playing indirect or complementary roles in some studies with implications for MCI and dementia (e.g., Hashimoto et al., 2009; Martinez et al., 2009; Forlenza et al., 2010; Rowe et al., 2010). It is conceivable that these two genetic variants often associated with non-demented cognitive aging phenotypes may be sensitive to early cognitive changes associated with preclinical transition processes. To our knowledge, no previous study has investigated the potential genetic links to MCI of all three genes in the same sample, nor to the longitudinal study of MCI status stability (normal, impairment) or instability (emerging impairment).

We test two research questions. First, we use baseline data to investigate whether $A P O E, B D N F$, or COMT polymorphisms independently or interactively differentiate between not-impaired cognitively (NIC control group) and MCI adults. Second, using longitudinal data, we test whether $A P O E, B D N F$, and COMT polymorphisms are independently or interactively associated with stability classification. Specifically, we used the stable normal aging two-wave combination (i.e., NIC-to-NIC) as the comparison for testing genetic influences on two theoretically and clinically viable profiles of cognitive status stability: (a) the conversion or declining status profile (NIC-to-MCI) and (b) the stable and chronic status profile (i.e., MCI-to-MCI).

\section{MATERIALS AND METHODS PARTICIPANTS}

This research was conducted under full, active, and continuous human ethics approval from prevailing Institutional Review Boards. Written informed consent was obtained from all participants. Participants were community-dwelling older adults from the Victoria Longitudinal Study (VLS), originally recruited through advertisements in the public media and to community groups. The VLS is an ongoing multi-sample sequential investigation of multiple aspects (e.g., cognitive, neuropsychological, health, sensory, and biological) of human aging. Detailed background information on the design, measures, and procedures is available (e.g., Dixon and de Frias, 2004). For this study, we assembled a 2 -wave (mean re-test interval $=4.59$ years) longitudinal data set by combining data collected during the same time period across VLS Samples 1 and 2, including only participants with genetic data. Specifically, the current Wave 1 (W1) data were from VLS Sample 1 (Wave 5) and VLS Sample 2 (Wave 3). Similarly, the current Wave 2 (W2) data were from VLS Sample 1 (Wave 6) and VLS Sample 2 (Wave 4). Data were analyzed first using only W1 data (cross-sectional) and then using the full 2-wave longitudinal design. At initial intake, VLS exclusionary criteria are implemented for all samples in order to establish relatively healthy cohorts of older adults, most of which proceed to develop aging-related cognitive and physical health conditions (Dixon and de Frias, 2004). For the present study, exclusionary criteria at baseline (W1) included a history of AD or any dementia, psychiatric disturbance or use of psychiatric medication, MMSE scores less than 24, serious cardiovascular or cerebrovascular disease conditions, uncontrolled hypertension, Type 1 diabetes, or history of serious head injury.

The first research question required the W1 baseline sample. Of the $\mathrm{W} 1$ participants $(n=237), 136$ met the criteria (see below) for the NIC group (Age: $M=73.12, S D=5.25$; Gender: $64 \%$ women; Years of education: $M=15.21, S D=2.94)$ and 101 met the standard criteria for the initial MCI group (Age: $M=$ 73.75, $S D=5.55$; Gender: 59.4\% women; Years of education: $M=14.52, S D=3.08)$. The second research question required two-wave longitudinal data. At W2, $n=218$ participants (of the 237) returned for testing (return rate $=92 \%$ ). We then excluded $n=1$ for missing scores on the cognitive status classification measures $(n=217)$. With two baseline status groups there were four possible two-wave status stability classifications. For the longitudinal analysis, we focused on the conceptually and clinically three most pertinent stability groups. First, the stable NIC (NIC-to-NIC: $n=101$; Age $M=73.23$, $S D=5.28$; Gender: $61.4 \%$ women; Years of Education $M=15.55, S D=$ 2.96) group was our benchmark (non-demented aging) comparison. Second, the corresponding declining NIC (NIC-to-MCI: $n=25$; Age $M=72.64, S D=5.30$; Gender: $72 \%$ women; Years of Education: $M=13.92, S D=2.12$ ) group represented status transition (emerging conversion, early MCI) over two waves. Third, the stable MCI (MCI-to-MCI: $n=68$; Age: $M=73.50$, $S D=5.40$; Gender: $55.9 \%$ women; Years of Education: $M=$ $14.32, S D=3.00)$ group represented continuing and chronic MCI status. Regarding the fourth (or "reversion;" Koepsell and Monsell, 2012) group, although previously observed in the literature (Palmer et al., 2002; Abner et al., 2012; Brodaty et al., 2013), we had no theory-based prediction for it. Therefore, for this and statistical reasons, we classified the MCI-to-NIC group ( $n=23$; Age $M=73.83, S D=5.57$; Gender: $65.2 \%$ women; Years of Education: $M=14.30, S D=3.13$ ) but did not include it in the analyses.

\section{COGNITIVE STATUS CLASSIFICATION PROCEDURE}

We used a standard and fully objective (non-clinical and nonsubtyped), four-step classification procedure, applying it independently at $\mathrm{W} 1$ and $\mathrm{W} 2$. The systematic procedure has been used in previous VLS studies and is consistent with other research and consensus reports (e.g., Ritchie et al., 2001; Winblad et al., 2004; Dixon et al., 2007; de Frias et al., 2009; Albert et al., 2011; Dolcos et al., 2012; Dixon and de Frias, 2014). The participants represented the complete subset available for genotyping of a somewhat larger VLS study of MCI (Dolcos et al., 2012). The larger sample was from the same population base and thus was used to optimize normative classification for the present subset (see Dolcos et al., 2012; $n=416$ at W1; $n=301 \mathrm{~W} 1-\mathrm{W} 2$ 
returnees). The VLS MCI classification procedure emphasized objective assessments of cohort-relative performance on a set of five cognitive reference measures, as evaluated in four successive steps. The first step is to stratify the sample by both age (64-73 and 74-95) and education (0-12 or 13+ years). The second step places each individual into one of four resulting age $\mathrm{x}$ education cells. The third step is to calculate mean and distributional characteristics for each of five cognitive reference measures. The five psychometrically sound tasks (e.g., Hultsch et al., 1998) represent the theoretical domains of perceptual speed, inductive reasoning, episodic memory, verbal fluency, and semantic memory. In the fourth step, these means and variability are used for withinsample norms and cognitive status classification. Specifically, we used a moderate criterion whereby participants were classified as MCI if they scored one or more standard deviations (SD) below their own age $\mathrm{x}$ education group means on one or more of the five cognitive reference tasks. The one SD criterion was previously established and represented an approach that provided a sensitive degree of differentiation appropriate to the present goal of detecting early signs of cognitive impairment (Ritchie et al., 2001; Dixon et al., 2007; de Frias et al., 2009; Dolcos et al., 2012). See description above and Table 1 for the results of the status classification.

\section{COGNITIVE REFERENCE MEASURES}

The five standard measures from the cognitive reference battery (e.g., Dixon et al., 2007; Dolcos et al., 2012) are widely available and documented with older adults. The psychometric properties and administrative details are documented and acceptable according to conventional standards (e.g., Hultsch et al., 1998). Performance at W1 for each subgroup is shown in Table 1.

\section{Perceptual speed}

Perceptual processing speed was assessed with the Wechsler Adult Intelligence Scale-Revised Digit Symbol Substitution (DSS) task (Wechsler, 1981). Psychometric characteristics of the DSS are well-established in aging and other populations (e.g., MacDonald et al., 2003). The number of correctly completed items was used as the final outcome.

\section{Inductive reasoning}

Inductive reasoning was assessed with the Letter Series test (Thurstone, 1962), frequently used with older adults. Participants were presented with 20 strings of letters forming a distinct pattern. The outcome was the total number correct.

\section{Episodic memory}

The VLS word recall task, consisting of immediate free recall of two lists of 30 English words selected from the total set of six equivalent lists (Dixon et al., 2004), was used. Each list consisted of six words from each of five taxonomic categories (e.g., birds, flowers), typed on a single page in unblocked order. The outcome was the average number of correctly recalled words.

\section{Verbal fluency}

We used the Controlled Associations test (synonyms) from the Educational Testing Service (ETS) kit of factor-referenced cognitive tests (Ekstrom et al., 1976). The outcome measure was the total number of correct synonyms.

Table 1 | Sample demographics at wave 1 by initial cognitive status at wave 1 and cognitive status stability (waves 1-2).

\begin{tabular}{|c|c|c|c|c|c|c|}
\hline Wave 1 & NIC & $\mathrm{MCl}$ & Wave 12 & NIC-NIC & NIC-MCI & MCl-MCl \\
\hline W1 & 136 & 101 & & 101 & 25 & $6 S$ \\
\hline W1 & $73.12(5.25)$ & $73.75(5.55)$ & & $73.23(5.28)$ & $72.64(5.30)$ & $73.50(5.40)$ \\
\hline \multicolumn{7}{|c|}{ GENDER (W) } \\
\hline W1 & $15.21(2.94)$ & $14.52(3.08)$ & & $15.55(2.96)$ & $13.92(2.12)$ & $14.32(3.00)$ \\
\hline \multicolumn{7}{|c|}{ PERCEPTUAL SPEED } \\
\hline W1 & $50.95(9.36)$ & $44.15(10.59)$ & & $51.58(9.89)$ & $49.52(7.69)$ & $45.19(10.10)$ \\
\hline \multicolumn{7}{|c|}{ INDUCTIVE REASONING } \\
\hline W1 & $12.99(3.38)$ & $9.31(4.83)$ & & $13.34(3.45)$ & $12.00(2.86)$ & $8.74(4.79)$ \\
\hline \multicolumn{7}{|c|}{ VOCABULARY } \\
\hline W1 & $46.26(3.90)$ & $42.14(5.56)$ & & $46.41(3.78)$ & $45.48(4.10)$ & $41.32(5.74)$ \\
\hline
\end{tabular}

W1, Wave 1; W2, Wave 2; Wave 12, Wave 1-Wave 2; N, Sample size; NIC, Not impaired controls; MCl, Mild cognitive impairment; Age and education data presented as Average (Standard Deviation): Cognitive domains represent the 5 cognitive reference measures used for cognitive status classification: For the two-wave groups (Wave 12), the data refer to baseline (W1) values. 


\section{Vocabulary}

The 54-item recognition, multiple-choice vocabulary test was composed by concatenating three 18-item tests from the ETS kit of factor referenced cognitive tests (Ekstrom et al., 1976). The total number of correct items representing the vocabulary score.

\section{DNA EXTRACTION AND GENOTYPING}

Following completion of the last testing session, saliva was collected according to standard procedures from Oragene DNA Genotek and stored at room temperature in Oragene ${ }^{\circledR}$ disks until DNA extraction. DNA was manually extracted from $0.8 \mathrm{ml}$ of saliva sample mix using the manufacturer's protocol with adjusted reagent volumes. Briefly, samples were incubated for $2.5 \mathrm{~h}$ at $50^{\circ} \mathrm{C}$ after inversion. Samples were transferred to a centrifuge tube and mixed with Oragene ${ }^{\circledR}$ purifier, incubated on ice for $10 \mathrm{~min}$, then centrifuged at $15,000 \mathrm{~g}$ for $5 \mathrm{~min}$ to pellet the denatured protein. The supernatant was transferred to a new tube and DNA was precipitated by adding an equal volume of $100 \%$ ethanol. The DNA pellet was washed with $70 \%$ ethanol, dried, and re-suspended with $10 \mathrm{mM}$ Tris, $\mathrm{pH}$ 8.0; $1 \mathrm{mM}$ EDTA buffer. DNA was incubated at $50^{\circ} \mathrm{C}$ for $1 \mathrm{~h}$ with occasional vortexing followed by incubation at $4^{\circ} \mathrm{C}$ overnight to ensure complete rehydration before quantification using a NanoDrop ${ }^{\circledR}$ ND-1000 Spectrophotometer (Wilmington, DE).

Genotyping was carried out with a Polymerase Chain Reaction (PCR) and Restricted Fragment Length Polymorphism (RFLP) strategy to analyze the allele status for APOE (determined by the combination of the Single Nucleotide Polymorphisms (SNPs) rs429358 and rs7412), BDNF (rs6265), and COMT (rs4680). SNP-containing PCR fragments were amplified in $25 \mathrm{ul}$ of $1 \mathrm{X}$ PCR reaction mix containing $25 \mathrm{ng}$ genomic DNA, $12.5 \mathrm{pmol}$ of each specific primer (APOE Forward: 5'-GGCACGGCTGT CCAAGGA-3'. APOE Reverse: 5'-GCCCCGGCCTGGTACACT GCC-3'; BDNF Forward: 5'-AAACATCCGAGGACAAGGTG-3'. BDNF Reverse: 5'-AGAAGAGGAGGCTCCAAAGG-3'; COMT Forward: 5'-GGGCCTACTGTGGCTACTCA-3'. COMT Reverse: $5^{\prime}$-CCCTTTTTCCAGGTCTGACA-3' $), 6.25 \mathrm{nmol}$ of each dNTP, 1.25U Taq DNA polymerase (New England Biolabs, NEB), $1.5 \mathrm{mM} \mathrm{MgCl} 2$ and $10 \%$ dimethylsulfoxide (DMSO). Reactions were set up in 96-well plates using the QIAgility robotic system (QIAgen). Specific amplicons were amplified using a program consisting of: denaturation step at $95^{\circ} \mathrm{C}$ for $2 \mathrm{~min}, 40$ cycles at $94^{\circ} \mathrm{C}$ for $30 \mathrm{~s}, 56^{\circ} \mathrm{C}$ for $30 \mathrm{~s}$, and $72^{\circ} \mathrm{C}$ for $1 \mathrm{~min}$, and then a final extension at $72^{\circ} \mathrm{C}$ for $7 \mathrm{~min}$. RFLP analysis was performed after digestion of the PCR amplicons with restriction enzymes (all from NEB) as follows: (a) APOE, for $16 \mathrm{~h}$ at $37^{\circ} \mathrm{C}$ with $\mathrm{HhaI}$, and (b) BDNF and COMT for $16 \mathrm{~h}$ at $37^{\circ} \mathrm{C}$ with NlaIII. RFLP analysis was then performed on a high resolution DNA screening cartridge on a QIAxcel capillary electrophoresis system (QIAgen) with the protocol OL700. The migration of the restriction fragments on 10 or $15 \%$ acrylamide gels for each SNP confirmed the analysis.

\section{DATA ANALYSIS}

All statistical analyses were performed using SPSS version 17.0 statistical software. For the W1 analysis, we used logistic regression to examine whether $A P O E, C O M T$, and BDNF polymorphisms were associated with initial cognitive group status (NIC vs. MCI). For the longitudinal analyses, we used multinomial logistic regression to examine whether $A P O E, B D N F$, and $C O M T$ polymorphisms were associated with stability of cognitive functioning (stable NIC-to-NIC, unstable NIC-to-MCI, and stable MCI-to-MCI) across the retest interval. In addition to standard independent candidate gene analyses, we also tested whether genetic risk would be magnified in the context of risk alleles combined across two cognitive-related genes. Because of limited cell sizes and power (in some instances) we followed the procedure of computing the two-way interactions, each of which compared the presence of both risk alleles/genotypes with all other possible genetic combinations for those particular genes. The two-way interactions between the test alleles ( $A P O E \varepsilon 4+, C O M T$ G/G, and BDNF A/A) were computed, as referenced by the three control alleles (APOE $\varepsilon 4-$, COMT A/A, and BDNF G/G). We assessed whether these genetic risk factors in two-way combinations (only) increased the risk of (a) MCI classification at baseline and (b) two-wave conversion to MCI (NIC-to-MCI) or MCI chronicity (MCI-to-MCI). Given our a priori directional hypotheses, we employed onetailed tests. Post-hoc logistic regression power analyses (GPower 3.1) indicate that the present study has (a) sufficient power to detect independent (genotype) effects (baseline single-factor power, $M=0.90$; longitudinal single-factor power, $M=0.80$ ), but (b) lower power to detect two-way (BDNF, COMT, APOE) interactions (baseline power, $M=0.62$; longitudinal power, $M=0.42$ ). We reported our interactive analyses cautiously, given few prior relevant reports using these polymorphisms for MCI.

\section{RESULTS}

We first report the initial allelic frequency results. For the two research questions age and education were entered into all analyses as covariates. Analyses without education as a covariate (Luciano et al., 2010) showed identical patterns.

\section{GENOTYPE AND ALLELIC FREOUENCIES}

Consistent with literature indicating that $A P O E, B D N F$, and $C O M T$ polymorphisms may be associated with lower levels of cognitive functioning in older adults (Bruder et al., 2005; Payton, 2009; Cathomas et al., 2010; Wisdom et al., 2011; Laukka et al., 2013), we informally expected that these genetic polymorphisms would show differential frequencies between the two cognitive status groups at baseline and among the four cognitive status stability groups (Brainerd et al., 2011). As can be seen in Table 1, participant demographics did not differ appreciably between waves or groups. Genotype and allele frequencies at baseline and at follow-up are reported in Table 2 (W1) and Table 3 (W1-W2) for each cognitive status and stability group. As seen in these tables, the distributions are reasonable and generally expected. For all three polymorphisms the W1 sample distributions were in Hardy-Weinberg equilibrium: (a) $\operatorname{COMT}\left(\chi^{2}=0.06\right.$, n.s. $)$, (b) BDNF ( $\chi^{2}=0.09$, n.s.), and (c) APOE (calculated in three groups rendered in terms of presence/absence of the $\varepsilon 4$ allele) $\left(\chi^{2}=0.02\right.$, n.s. $)$. 
Table 2 | Frequency of $A P O E, C O M T$ and $B D N F$ genotypes and alleles at wave 1 , as stratified by cognitive status.

\begin{tabular}{lcc}
\hline Variable & NIC & MCl \\
\hline N & 136 & 101 \\
APOE & $106(77.9)$ & $69(68.3)$ \\
No $\varepsilon 4$ & $30(22.1)$ & $32(31.7)$ \\
$\geq 1 \varepsilon 4$ & & \\
COMT & $31(22.8)$ & $20(19.8)$ \\
A/A & $80(58.8)$ & $53(52.5)$ \\
A/G & $25(18.4)$ & $28(27.7)$ \\
G/G & $8(5.9)$ & $4(4)$ \\
BDNF & $36(26.5)$ & $34(33.7)$ \\
A/A & $92(67.6)$ & $63(62.4)$ \\
A/G & & \\
G/G & . & \\
\hline
\end{tabular}

APOE, Apolipoprotein E; $\varepsilon 4, A P O E$ epsilon 4 allele; BDNF, Brain derived neurotrophic factor; COMT, Catechol O-methyltransferase; $G$, Guanine, Valine amino acid; A, Adenine, Methionine amino acid; N, Sample size; NIC, Not Impaired Controls; $\mathrm{MCl}$, Mild Cognitive Impairment; $\geq 1 \varepsilon 4$, the presence of at least 1 $A P O E \& 4$ allele; data presented as Frequency (Percentage).

\section{GENETIC MARKERS OF BASELINE COGNITIVE STATUS}

We used binary logistic regression to examine baseline group differences in (a) cognitive status (NIC vs. MCI) and (b) APOE, COMT and BDNF genotype. As reported in Table 4, the presence of at least one $A P O E \varepsilon 4$ allele was associated with a 1.65 -fold higher likelihood of MCI classification at baseline compared to NIC $[p=0.047$ (1-tailed)]. The COMT A/G (Met/Val) or COMT G/G (homozygous Val) genotypes were not associated with an increased risk of MCI classification at baseline compared to the COMT A/A (homozygous Met) genotype. Similarly, the BDNF $\mathrm{A} / \mathrm{G}$ or BDNF A/A (homozygous Met) genotypes were not associated with an increased risk of MCI classification at baseline compared to the BDNF G/G (homozygous Val) genotype. The 2way interactions between $A P O E, C O M T$, and BDNF revealed no significant increased risk of MCI classification at baseline for the interaction terms.

\section{GENETIC MARKERS OF TWO-WAVE STABILITY OF COGNITIVE STATUS}

The association between APOE, COMT, and BDNF genotype with cognitive status stability across the W1-W2 retest interval was examined using multinomial logistic regression. Consistent with previous reports which indicate that $A P O E, B D N F$, and COMT polymorphisms may be implicated in cognitive decline (Bruder et al., 2005; Blom et al., 2009; Hashimoto et al., 2009; Schiepers et al., 2012), we expected that APOE, BDNF, and COMT polymorphisms would be associated with NIC instability (i.e., emerging impairment, NIC-to-MCI) and MCI stability (i.e., impairment chronicity, MCI-to-MCI) across the retest interval. As reported in Table 4, the presence of at least one APOE $\varepsilon 4$ allele was associated with a 1.82-fold higher likelihood of MCI-to-MCI stability (chronicity) over time compared to a stable NIC-to-NIC classification ( $p=0.050,1$-tailed). Although $A P O E \& 4$ was not associated with NIC-to-MCI decline over time, a dose-response effect for the $\varepsilon 4$ allele was noted between
Table 3 | Frequency of APOE, COMT and BDNF genotypes and alleles across the W1-W2 retest interval, as stratified by cognitive status stability group.

\begin{tabular}{lcccc}
\hline Variable & Wave & NIC-NIC & NIC-MCI & MCI-MCl \\
\hline $\boldsymbol{N}$ & W2 & 101 & 25 & 68 \\
APOE & & & & \\
no $\varepsilon 4$ & W2 & $80(79.2)$ & $18(72)$ & $46(67.6)$ \\
$\geq 1 \varepsilon 4$ & W2 & $21(20.8)$ & $7(28)$ & $22(32.4)$ \\
COMT & & & & \\
A/A & W2 & $27(26.7)$ & $4(16)$ & $14(20.6)$ \\
A/G & W2 & $59(58.4)$ & $13(52)$ & $35(51.5)$ \\
G/G & W2 & $15(14.9)$ & $8(32)$ & $19(27.9)$ \\
BDNF & & & & \\
A/A & W2 & $5(5)$ & $2(8)$ & $3(4.4)$ \\
A/G & W2 & $29(28.7)$ & $4(16)$ & $20(29.4)$ \\
G/G & W2 & $67(66.3)$ & $19(76)$ & $45(66.2)$ \\
\hline
\end{tabular}

APOE, Apolipoprotein E; $\varepsilon 4$, APOE epsilon 4 allele; BDNF, Brain Derived Neurotrophic Factor; COMT, Catechol O-methyltransferase; G, Guanine, Valine amino acid; A, Adenine, Methionine amino acid; W1, Wave 1; W2, Wave 2; $N$, Sample size; NIC, Not Impaired Controls; MCl, Mild Cognitive Impairment; $\geq 1$ $\varepsilon 4$, the presence of at least 1 APOE $\varepsilon 4$ allele; data presented as Frequency (Percentage).

the 2-wave cognitive stability groups (i.e., NIC-to-NIC, MCIto-MCI; Table 4). Although the COMT G/G (homozygous Val) genotype was not associated with baseline status, it was associated with both a 3.94-fold higher likelihood of NIC-to-MCI decline and a 2.60-fold higher likelihood of MCI-to-MCI stability (chronicity) over time, as compared to the stable NIC-to-NIC classification group ( $p=0.027$ and 0.025 , respectively, 1 -tailed). COMT A/G was not associated with either two-wave profile. The BDNF A/A (homozygous Met) or A/G genotypes were not associated with NIC-to-MCI conversion or with MCI-to-MCI stability over time. The 2-way interactions between APOE, BDNF, and COMT did not confer any significant associations with the stability and status groups.

\section{DISCUSSION}

We examined risk of objectively assessed MCI (distinguished from non-demented aging) as associated with risk alleles of three relevant polymorphisms. Group membership in three MCI profiles was examined: (a) baseline (W1), with NIC compared with MCI, (b) two-wave stability of MCI status, reflecting chronic and stable condition (i.e., MCI-to-MCI), and (c) two-wave decline in NIC status, reflecting early and emerging conversion to classifiable impairment (i.e., NIC-to-MCI). This study both replicates and extends recent single-wave MCI assessments by including (a) two-wave independent follow-up classifications, (b) tests of the typical APOE genetic variant plus two additional variants, and (c) assessments of two-wave patterns reflecting impaired chronicity and emerging conversion.

We discuss the results as organized by the three genetic polymorphisms. First, $A P O E$ was expected to be a significant marker. The $\varepsilon 4$ variant is the best known genetic risk factor for sporadic 
Table 4 | Statistical results relating genetic risk factors to cognitive status and stability for APOE, COMT and BDNF.

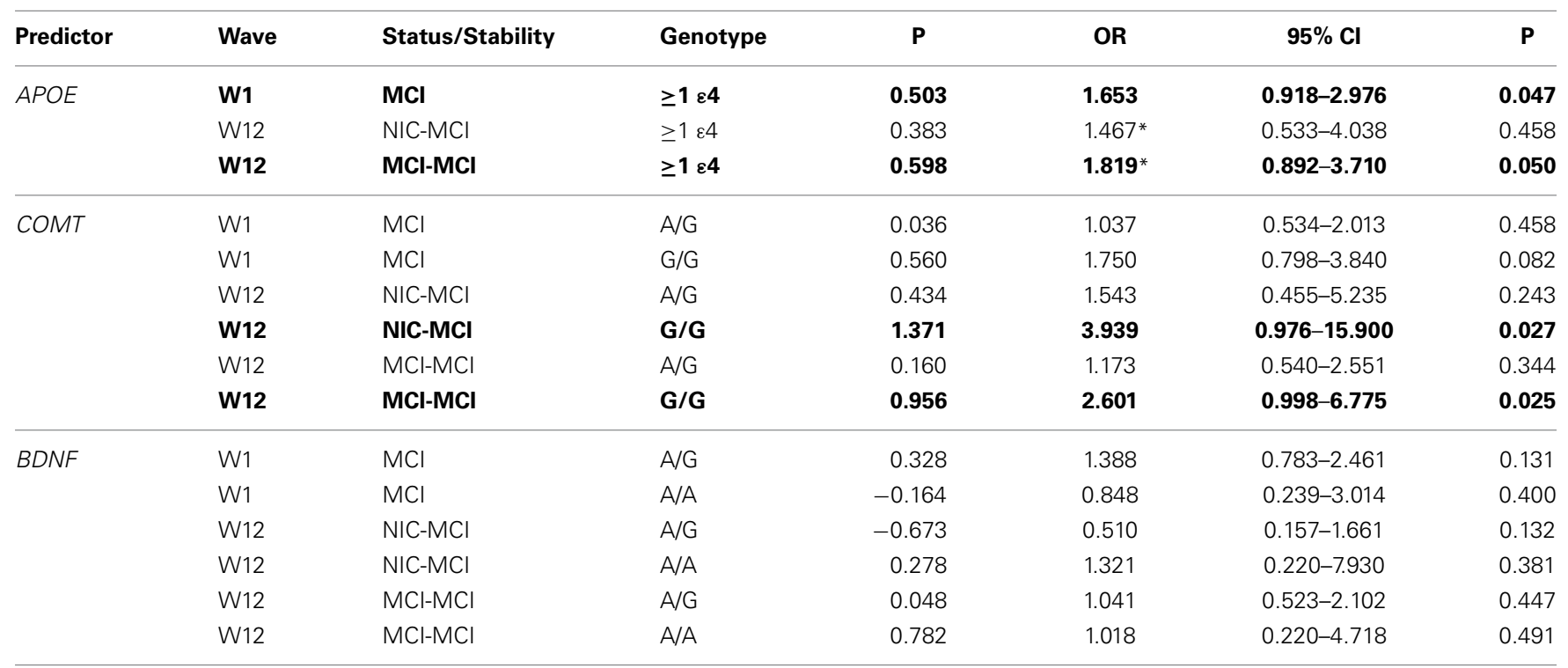

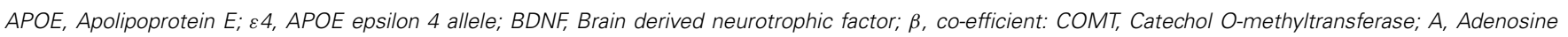
nucleotide, Methionine amino acid; G, Guanine nucleotide, Valine amino acid; N, sample size; NIC, Not Impaired Controls; MCl, Mild Cognitive Impairment; OR, odds ratio: W1, Wave 1; W12, Wave 1-Wave 2; $\geq 1 \varepsilon 4$, the presence of at least 1 APOE s4 allele; for APOE analyses, the presence of $\geq 1 \varepsilon 4$ was compared to the

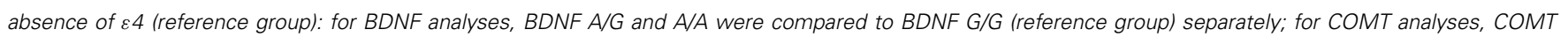

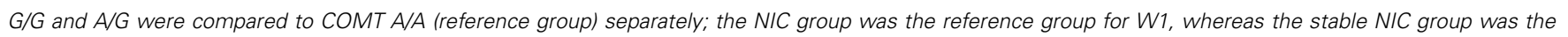
reference group for W1-W2 analyses; $p$ values are all presented as one-tailed; stars indicate dose response findings.

AD (Albert et al., 2011; Harris and Deary, 2011) and has been implicated in candidate gene studies for normal cognitive decrements (e.g., Small et al., 2004; Kozauer et al., 2008; Liu et al., 2010; Izaks et al., 2011; Wisdom et al., 2011; Davies et al., 2014) and mild cognitive impairment (Brainerd et al., 2011; Reinvang et al., 2013). Brainerd and colleagues reported that the $\varepsilon 4$ variant was more represented in older adults classified as MCI than in a nondemented comparison group. Our results are consistent with (and extend) these findings. Using fully objective classification procedures, we observed that the presence of at least one APOE \&4 allele conferred increased risk of MCI classification (a) at baseline and (b) for stability (chronicity) over the two-wave interval. We interpret the latter as evidence both for the stability of our MCI classifications and for their usefulness for detecting preclinical decrements early in cognitive aging. Notably, we use the objective, multi-dimensional cognitive reference battery approach to capture mild disorder that (a) may be transient or variable among some such early cases of impairment (Koepsell and Monsell, 2012), (b) may even be reflected by modest deficits (i.e., 1 SD) in a single fundamental domain (Dolcos et al., 2012), and (c) do not reflect the potential that differential domain deficits may be indicative across waves and subtypes (Brodaty et al., 2013). For this reason, the classification battery represents five complementary domains of basic cognitive resources. Within the objective VLS classification approach, this is the first evidence regarding genetic associations, but it has previously pointed to MCI associations with executive functioning (de Frias et al., 2009), memory (Dixon and de Frias, 2014), neurocognitive inconsistency (Dixon et al., 2007), functional biomarkers (Dolcos et al., 2012), and molecular-level metabolomics markers (Zheng et al.,
2012). Future progress will be achieved when researchers are able to assemble and analyze multifaceted sets of markers relevant to early MCI transitions, stability, and outcomes (Fotuhi et al., 2009; Anstey, 2014). Although we present two-wave data, we must await future waves to discover the extent to which MCI-to-MCI chronicity is also probabilistically linked to eventual AD. For now, the predictions of surrogate outcomes (MCI baseline status and 4-year stability or chronicity) are promising results.

Second, the COMT G variant (including Val-Val and Val-Met) has been identified as a risk factor for cognitive deficits with normal aging, given the association with the degradation of catecholamine neurotransmitters (such as dopamine) (Savitz et al., 2006). However, previous results of candidate gene studies have revealed promising but inconsistent association patterns (Barnett et al., 2008; Wishart et al., 2011; Sapkota et al., 2014) and MCI status (Martinez et al., 2009; Yarnell et al., 2014). Had we only examined concurrent data we would have reported no MCI-related associations. Instead, the COMT variant emerged as a significant marker of MCI chronicity and transition in the two-wave data. This may be due to its sensitivity to early cognitive aging disorders in performances represented by the present broad-based neurocognitive reference battery, especially among older adults who are transitioning (NIC-to-MCI) or chronically MCI. These dynamic groups may present more homogeneous MCI-related phenotypes than the larger baseline source group. The extent to which the COMT G variant (homozygous $\mathrm{G} / \mathrm{G}$ ) was involved in predicting status chronicity and conversion was novel and promising. Together with recent work showing promising synergistic effects (Martinez et al., 2009; Lanni et al., 2012), COMT may deserve additional attention in MCI, especially in the context 
of $A P O E$, longitudinal follow-ups, and phenotypes reflecting its neurobiological influence. Perhaps with objective but sensitive assessment procedures, COMT may prove to be a candidate genetic susceptibility marker of early cognitive changes associated with neurobiological disruptions that may be secondary or preliminary to AD-related neuropathology. At present, the absence of COMT from the pantheon of MCI-related genes may be associated with the fact that, by the time individuals convert to dementia, the genetic associations are overwhelmed by APOE and its direct connection to amyloid beta deposition. Future research can investigate whether the apparent separate contributions of $A P O E$ and COMT to MCI status and change continue to be complementary or whether a gene-related would produce synergistic vulnerability (Lindenberger et al., 2008; McFall et al., 2014).

Third, the other polymorphism $(B D N F)$ was not statistically associated with the targeted outcomes. In general, BDNFcognition associations are inconsistently observed and difficult to interpret (Mandelman and Grigorenko, 2012). In addition, BDNF associations are rarely tested as predictors of clinical or cognitive status or neurodegenerative changes (cf. Forlenza et al., 2010). The mechanisms through which BDNF may affect neurocognitive performance are proposed (Savitz et al., 2006; Harris and Deary, 2011) but their relevance to non-normal cognitive status has not been firmly established. As an MCI predictor, BDNF may be less relevant for early classification (as in this study) than for later cognitive impairment (Forlenza et al., 2010). We included $B D N F$ for two reasons: (a) it could have been related to objective cognitive status as we assess it (via performance on a reference battery including memory and speed markers) and (b) it could have appeared as an associate of cognitive status in the role of interacting influence.

Overall, the findings of this study reveal several interesting independent and complementary genetic associations with non-demented aging and MCI status, including initial, transitioning, and chronic profiles. However, several limitations should be acknowledged. First, not all VLS data were available for the present study, as the first phase of the genetics initiative occurred only recently, thus limiting the overall and (especially) clinical group sample sizes. Further genotyping and follow-up waves are planned. Second, our MCI classification procedures are derived from consensus statements (e.g., Albert et al., 2011) and standardized procedures (e.g., Dixon et al., 2007). They do not include several characteristics sometimes observed in the literature: (a) clinical judgment or personal evaluations, (b) differentiation into subtypes of MCI, or (c) criteria emphasizing established or severe pre-dementia cases (e.g., Winblad et al., 2004; Petersen and Knopman, 2006; Dolcos et al., 2012). Instead, we emphasized (a) replicable and transferable classification (i.e., objective procedures may be translated into multiple research and clinical settings), (b) overall MCI classification based on deficits in multiple possible basic domains as based on the assumption that early or emergent cases may not (yet) be consolidated into subtypes, and (c) use of moderate criteria (i.e., 1 SD below the appropriate group mean) so that early cases may be identified, at the risk of some false positives. In the VLS, transitions into MCI and dementia are slowly emerging as the VLS itself "ages." Emergence of differential subtypes, etiology, and severities will be followed, as they could modify future interpretations. Third, we focus on predictors of emergence, stability, and early transitions in MCI. Extending research to predictors of later transitions (e.g., MCI-dementia) will require larger samples of well-diagnosed dementia patients. Other researchers are also embracing the challenge of investigating characteristics and markers of early MCI (e.g., Kryscio et al., 2006; Anstey et al., 2013; Risacher et al., 2013) using status change or stability as surrogate outcomes.

Fourth, the sample size of the present study was limited because the design required participation in two longitudinal waves. Relevant published studies vary in sample size, age ranges, and availability of longitudinal intervals (Caselli et al., 2007, 2014; Martinez et al., 2009; Forlenza et al., 2010; Brodaty et al., 2013; Risacher et al., 2013). We reported a limitation in the power to detect gene $\mathrm{x}$ gene interactions. However, the fact that we did not observe significant synergistic interactions may also be related to other fundamental aspects of the emergence and progression of relatively early MCI in aging: (a) these processes may not have been sufficiently advanced to "recruit" or "require" such interactional contributions, (b) these genetic variants may not be those that are involved in interactional predictions of MCI, and (c) more diverse, more select, more severe, or etiologically purer samples might extend the present results. Much larger studies may be better powered for interaction analyses, but this can balanced if they lack the nuanced or valid cognitive measures or have suboptimal intervals. Therefore, we do not rule out the potential role of gene $\mathrm{x}$ gene interactions in predicting the phenomena we address in this study. Fifth, there are a number of unmeasured factors in any study of normal cognitive aging or emergent and longitudinal MCI phenomena (e.g., Fotuhi et al., 2009; Albert et al., 2011; Woodard et al., 2012). One possible avenue for future research includes investigation of multiple AD-related markers (Risacher et al., 2013) or gene x environment (e.g., health; Anstey, 2014; McFall et al., 2014) effects. Sixth, we studied only three genetic variants, and others could be investigated in the prediction of MCI status, stability, and transitions. However, many candidate gene studies of MCI include only one variant (typically $A P O E$ ). We recommend the careful selection of additional polymorphisms based on known underlying neurobiological mechanisms related to $\mathrm{MCI}$.

In sum, we observed two sets of independent and complementary associations with theoretically and clinically relevant phenomena of early cognitive impairment in aging. Both early and more advanced cognitive impairment is likely the result of a complex, life-long series of interactions among various genetic susceptibility and environmental risk factors. Regarding genetic polymorphisms, both APOE and COMT hold promise for future complex and dynamic analyses of the short- and long-term processes through which some normally aging individuals begin transitioning into preclinical impairment.

\section{ACKNOWLEDGMENTS}

This research was supported by grants from (a) the National Institutes of Health (National Institute on Aging; R01 AG008235) to author Roger A. Dixon, (b) the Alberta Health Services and the University Hospital Foundation (to authors RAD, JJ, DW), (c) the Canada Research Chairs program (to authors RAD 
and DW), and (d) the Michael Smith Foundation for Health Research (Scholar Award) to author SM. This research could not have taken place without the dedicated VLS participants, research assistants, and postdocs (including Sanda Dolcos, Inka Fakuade, Jill Friesen, Terry Perkins, and Bonnie Whitehead). Further information about the VLS may be accessed via: http://www.ualberta.ca/ vlslab/index.html. Contact Dr. Dixon at: rdixon@ualberta.ca.

\section{REFERENCES}

Abner, E. L., Kryscio, R. J., Cooper, G. E., Fardo, D. W., Jicha, G. A., Mendiondo M. S., et al. (2012). Mild cognitive impairment: statistical models of transition using longitudinal clinical data. Int. J. Alzheimers Dis. 2012:291920. doi: $10.1155 / 2012 / 291920$

Albert, M. S., DeKoskey, S. T., Dickson, D., Dubois, B., Feldman, H. H., Fox, N. C., et al. (2011). The diagnosis of mild cognitive impairment due to Alzheimer's disease. Alzheimers Dement. 7, 270-279. doi: 10.1016/j.jalz.2011.03.008

Anstey, K. J. (2014). Optimizing cognitive development over the life course and preventing cognitive decline: introducing the Cognitive Health Environment Life Course Model (CHELM). Int. J. Behav. Dev. 38, 1-10. doi: $10.1177 / 0165025413512255$

Anstey, K. J., Cherbuin, N., Eramudugolla, R., Sargent-Cox, K., Easteal, S., Kumar, R., et al. (2013). Characterizing mild cognitive disorders in the youngold over 8 years: prevalence, estimated incidence, stability of diagnosis, and impact on IADLs. Alzheimers Dement. 9, 640-648. doi: 10.1016/j.jalz.2012. 11.013

Barabash, A., Marcos, A., Ancín, I., Vazquez-Alvarez, B., de Ugarte, C., Gil, P., et al. (2009). APOE, ACT and CHRNA7 genes in the conversion from amnestic mild cognitive impairment to Alzheimer's disease. Neurobiol. Aging 30, 1254-1264. doi: 10.1016/j.neurobiolaging.2007.11.003

Barnett, J. H., Scoriels, L., and Munafo, M. R. (2008). Meta-analysis of the cognitive effects of the Catechol-O-methyltransferase gene Val158/108Met polymorphism. Biol. Psychiatry 64, 137-144. doi: 10.1016/j.biopsych.2008. 01.005

Bertram, L., McQueen, M. B., Mullin, K., Blacker, D., and Tanzi, R. E. (2007). Systematic meta-analyses of Alzheimer disease genetic association studies: the AlzGene database. Nat. Genet. 39, 17-23. doi: 10.1038/ng1934

Blom, E. S., Giedraitis, V., Zetterberg, H., Fukumoto, H., Blennow, K., Hyman, B. T., et al. (2009). Rapid progression from mild cognitive impairment to Alzheimer's disease in subjects with elevated levels of tau in cerebrospinal fluid and the APOE epsilon4/epsilon4 genotype. Dement. Geriatr. Cogn. Disord. 27, 458-464. doi: 10.1159/000216841

Brainerd, C. J., Reyna, V. F., Petersen, R. C., Smith, G. E., and Taub, E. S. (2011). Is the Apolipoprotein e genotype a biomarker for mild cognitive impairment? Findings from a nationally representative study. Neuropsychology 25, 679-689. doi: $10.1037 / \mathrm{a} 0024483$

Brodaty, H., Heffernan, M., Kochan, N. A., Draper, B., Trollar, J. N., Reppermund, S., et al. (2013). Mild cognitive impairment in a community sample: the Sydney memory and ageing study. Alzheimers Dement. 9, 310-317. doi: 10.1016/j.jalz.2011.11.010

Bruder, G. E., Keilp, J. G., Xu, H., Shikhman, M., Schori, E., Gorman, J. M., et al. (2005). Catechol-O-methyltransferase (COMT) genotypes and working memory: associations with differing cognitive operations. Biol. Psychiatry 58, 901-907. doi: 10.1016/j.biopsych.2005.05.010

Caselli, R. J., Locke, D. E. C., Dueck, A. C., Knopman, D. S., Woodruff, B. K., Hoffman-Snyder, C., et al. (2014). The neuropsychology of normal aging and preclinical Alzheimer's disease. Alzheimers Dement. 10, 84-92. doi: 10.1016/j.jalz.2013.01.004

Caselli, R. J., Reiman, E. M., Locke, D. E., Hutton, M. L., Hentz, J. G., HoffmanSnyder, C., et al. (2007). Cognitive domain decline in healthy apolipoprotein E epsilon4 homozygotes before the diagnosis of mild cognitive impairment. Arch. Neurol. 64, 1306-1311. doi: 10.1001/archneur.64.9.1306

Cathomas, F., Vogler, C., Euler-Sigmund, J. C., de Quervain, D. J., and Papassotiropoulos, A. (2010). Fine-mapping of the Brain-derived neurotrophic factor (BDNF) gene supports an association of the Val66Met polymorphism with episodic memory. Int. J. Neuropsychopharmacol. 13, 975-980. doi: $10.1017 /$ S1461145710000519
Davies, G., Harris, S. E., Reynolds, C. A., Payton, A., Knight, H. M., Liewald, D. C., et al. (2014). A genome-wide association study implicates the APOE locus in nonpathological cognitive aging. Mol. Psychiatry 19, 76-87. doi: 10.1038/mp. 2012.159

de Frias, C. M., Dixon, R. A., and Strauss, E. (2009). Characterizing executive functioning in older special populations: from cognitively elite to cognitively impaired. Neuropsychology 23, 778-791. doi: 10.1037/a0016743

Dixon, R. A., and de Frias, C. M. (2004). The victoria longitudinal study: from characterizing cognitive aging to illustrating changes in memory compensation. Aging Neuropsychol. Cogn. 11, 346-376. doi: 10.1080/138255804905 11161

Dixon, R. A., and de Frias, C. M. (2014). Cognitively elite, cognitively normal, and cognitively impaired aging: neurocognitive status and stability moderate memory performance. J. Clin. Exp. Neuropsychol. 36, 418-430. doi: $10.1080 / 13803395.2014 .903901$

Dixon, R. A., Garrett, D. D., Lentz, T. L., MacDonald, S. W. S., Strauss, E., and Hultsch, D. F. (2007). Neurocognitive markers of cognitive impairment: exploring the roles of speed and inconsistency. Neuropsychology 21, 381-399. doi: 10.1037/0894-4105.21.3.381

Dixon, R. A., Small, B. J., MacDonald, S. W. S., and McArdle, J. J. (2012). "Yes, memory declines with aging-but when, how, and why?," in Memory and aging, eds M. Naveh-Benjamin and N. Ohta (New York, NY: Psychology Press), 325-347.

Dixon, R. A., Wahlin, Å., Maitland, S. B., Hultsch, D. F., Hertzog, C., and Bäckman, L. (2004). Episodic memory change in late adulthood: generalizability across samples and performance indices. Mem. Cogn. 32, 768-778. doi: 10.3758/BF03195867

Dolcos, S., MacDonald, S. W. S., Braslavsky, A., Camicioli, R., and Dixon, R. A. (2012). Mild cognitive impairment is associated with selected functional markers: integrating concurrent, longitudinal, and stability effects. Neuropsychology 26, 209-223. doi: 10.1037/a0026760

Ekstrom, R. B., French, J. E. W., Harman, H. H., and Dermen, D. (1976). Manual for the Kit of Factor-Referenced Cognitive Tests. Princeton, NJ: Educational Testing Service.

Elias-Sonnenschein, L. S., Viechtbauer, W., Ramakers, I. H., Verhey, F. R., and Visser, P. J. (2011). Predictive value of APOE-epsilon4 allele for progression from MCI to AD-type dementia: a meta-analysis. J. Neurol. Neurosurg. Psychiatry 82, 1149-1156. doi: 10.1136/jnnp.2010.231555

Forlenza, O. V., Diniz, B. S., Teixeira, A. L., Ojopi, E. B., Talib, L. L., Mendonca, V. A., et al. (2010). Effect of Brain-derived neurotrophic factor Val66Met polymorphism and serum levels on the progression of mild cognitive impairment. World J. Biol. Psychiatry 11, 774-780. doi: 10.3109/15622971003797241

Fotuhi, M., Hachinski, V., and Whitehouse, P. J. (2009). Changing perspectives regarding late-life dementia. Nat. Rev. Neurol. 5, 649-658. doi: 10.1038/nrneurol.2009.175

Gauthier, S., Reisberg, B., Zaudig, M., Petersen, R. C., Ritchie, K., Broich, K., et al. (2006). Mild cognitive impairment. Lancet 367, 1262-1270. doi: 10.1016/S01406736(06)68542-5

Geda, Y. E., and Nedelska, Z. (2012). Mild cognitive impairment: a subset of minor neurocognitive disorder? Am. J. Geriatr. Psychiatry 20, 821-826. doi: 10.1097/JGP.0b013e31826abc00

Golde, T. E., Schneider, L. S., and Koo, E. H. (2011). Anti-A $\beta$ therapeutics in Alzheimer's disease: the need for a new paradigm shift. Neuron 69, 203-213. doi: 10.1016/j.neuron.2011.01.002

Harris, S. E., and Deary, I. J. (2011). The genetics of cognitive ability and cognitive ageing in healthy older people. Trends Cogn. Sci. 15, 388-394. doi: 10.1016/j.tics.2011.07.004

Hashimoto, R., Hirata, Y., Asada, T., Yamashita, F., Nemoto, K., Mori, T., et al. (2009). Effect of the Brain-derived neurotrophic factor and the Apolipoprotein E polymorphisms on disease progression in preclinical Alzheimer's disease. Genes Brain Behav. 8, 43-52. doi: 10.1111/j.1601-183X.2008. 00440.x

Hultsch, D. F., Hertzog, C., Dixon, R. A., and Small, B. J. (1998). Memory Change in the Aged. New York, NY: Cambridge University Press.

Hurd, M. D., Martorell, P., Delavande, A., Mullen, K. J., and Langa, K. M. (2013). Monetary costs of dementia in the United States. N. Engl. J. Med. 368, 1326-1334. doi: 10.1056/NEJMsa1204629

Izaks, G. J., Gansevoort, R. T., van der Knaap, A. M., Navis, G., Dullaart, R. P. F., and Slaets, J. P. J. (2011). The association of APOE genotype with 
cognitive function in persons aged 35 years or older. PLoS ONE 6:e27415. doi: 10.1371/journal.pone.0027415

Jorm, A. F., Mather, K. A., Butterworth, P., Anstey, K. J., Christensen, H., and Easteal, S. (2007). APOE genotype and cognitive functioning in a large age-stratified population sample. Neuropsychology 21, 1-8. doi: 10.1037/08944105.21.1.1

Josefsson, M., de Luna, X., Pudas, S., Nilsson, L.-G., and Nyberg, L. (2012). Genetic and lifestyle predictors of 15-year longitudinal change in episodic memory. J. Am. Geriatr. Soc. 60, 2308-2312. doi: 10.1111/jgs. 12000

Koepsell, T. D., and Monsell, S. E. (2012). Reversion from mild cognitive impairment to normal or near-normal cognition. Neurology 79, 1591-1598. doi: 10.1212/WNL.0b013e31826e26b7

Kozauer, N. A., Mielke, M. M., Chan, G. K. C., Rebok, G. W., and Lyketsos, C. G. (2008). Apolipoprotein E genotype and lifetime cognitive decline. Int Psychogeriatr. 20, 109-123. doi: 10.1017/S104161020700587X

Kryscio, R. J., Schmitt, F. A., Salazar, J. C., Mendiondo, M. S., and Markesbery, W. R. (2006). Risk factors for transitions from normal to mild cognitive impairment and dementia. Neurology 66, 828-832. doi: 10.1212/01.wnl.0000203264.71880.45

Lane, R., Feldman, H. H., Meyer, J., He, Y., Ferris, S. H., Nordberg, A., et al. (2008). Synergistic effect of Apolipoprotein E epsilon4 and Butyrylcholinesterase Kvariant on progression from mild cognitive impairment to Alzheimer's disease. Pharmacogenet. Genomics 18, 289-298. doi: 10.1097/FPC.0b013e3282 f63f 29

Lanni, C., Garbin, G., Lisa, A., Biundo, F., Ranzenigo, A., Sinforiani, E., et al. (2012). Influence of COMT Val58Met polymorphism on Alzheimer's disease and mild cognitive impairment in Italian patients. J. Alzheimers Dis. 32, 919-926. doi 10.1017/S104161020700587X

Laukka, E. J., Lövdén, M., Herlitz, A., Karlsson, S., Ferencz, B., Pantzar, A., et al. (2013). Genetic effects on old-age cognitive functioning: a population-based study. Psychol. Aging 28, 262-274. doi: 10.1037/a0030829

Lin, P.-J., and Neumann, P. J. (2013). The economics of mild cognitive impairment. Alzheimers Dement. 9, 58-62. doi: 10.1016/j.jalz.2012.05.2117

Lindenberger, U., Nagel, I. E., Chicherio, C., Li, S.-C., Keekeren, H. R., and Bäckman, L. (2008). Age-related decline in brain resources modulates genetic effects on cognitive functioning. Front. Neurosci. 2, 234-244. doi 10.3389/neuro.01.039.2008

Liu, F., Pardo, L. M., Schuur, M., Sanchez-Juan, P., Isaacs, A., Sleegers, K., et al. (2010). The Apolipoprotein E gene and its age-specific effects on cognitive function. Neurobiol. Aging 31, 1831-1833. doi: 10.1016/ j.neurobiolaging.2008.09.015

Luciano, M., Batty, G. D., McGilchrist, M., Linksted, P., Fitzpatrick, B., Jackson, C., et al. (2010). Shared genetic aetiology between cognitive ability and cardiovascular disease risk factors: generation Scotland's Scottish family health study. Intelligence 38, 304-313. doi: 10.1016/j.intell.2010.03.002

MacDonald, S. W. S., Hultsch, D. F., Strauss, E., and Dixon, R. A. (2003). Agerelated slowing of digit symbol substitution revisited: what do longitudinal age changes reflect? J. Gerontol. B Psychol. Sci. Soc. Sci. 58, P187-P194. doi: 10.1093/geronb/58.3.P187

Mandelman, S. D., and Grigorenko, E. L. (2012). BDNF Val66Met and cognition: All, none, or some? A meta-analysis of the genetic association. Genes Brain Behav. 11, 127-136. doi: 10.1111/j.1601-183X.2011.00738.x

Martinez, M. F., Martin, X. E., Alcelay, L. G., Flores, J. C., Valiente, J. M., Juanbeltz, B. I., et al. (2009). The COMT Val158 met polymorphism as an associated risk factor for Alzheimer disease and mild cognitive impairment in APOE 4 carriers. BMC Neurosci. 10:125. doi: 10.1186/1471-2202-10-125

McFall, G. P., Wiebe, S. A., Vergote, D., Jhamandas, J., Westaway, D., and Dixon, R. A. (2014). IDE (rs6583817) polymorphism and pulse pressure are independently and interactively associated with level and change in executive function in older adults. Psychol. Aging 29, 418-430. doi: 10.1037/a0034656

McKhann, G. M., Knopman, D. S., Chertkow, H., Hyman, B. T., Jack, C. R. Jr., Kawas, C. H., et al. (2011). The diagnosis of dementia due to Alzheimer's disease: recommendations from the national institute on Aging-Alzheimer's association workgroups on diagnostic guidelines for Alzheimer's disease. Alzheimers Dement. 7, 263-269. doi: 10.1016/j.jalz.2011.03.005

Miyajima, F., Ollier, W., Mayes, A., Jackson, A., Thacker, N., Rabbitt, P., et al. (2008). Brain-derived neurotrophic factor polymorphism Val66Met influences cognitive abilities of the elderly. Genes Brain Behav. 7, 411-417. doi: 10.1111/j.1601183X.2007.00363.x
Palmer, K., Wang, H. X., Bäckman, L., Winblad, B., and Fratiglioni, L. (2002). Differential evolution of cognitive impairment in nondemented older persons: results from the Kungsholmen Project. Am. J. Psychiatry 159, 436-442. doi: 10.1176/appi.ajp.159.3.436

Payton, A. (2006). Investigating cognitive genetics and its implications for the treatment of cognitive deficit. Genes Brain Behav. 5(Suppl. 1), 44-53. doi: 10.1111/j.1601-183X.2006.00194.X

Payton, A. (2009). The impact of genetic research on our understanding of normal cognitive ageing: 1995 to 2009. Neuropsychol. Rev. 19, 451-477. doi: 10.1007/s11065-009-9116-z

Petersen, R. C., and Knopman, D. S. (2006). MCI is a clinically useful concept. Int. Psychogeriatr. 18, 394-402. doi: 10.1017/S1041610206003929

Plassman, B. L., Williams, J. W., Burke, J. R., Holsinger, T., and Benjamin, S. (2010). Systematic review: factors associated with risk for and possible prevention of cognitive decline in later life. Ann. Intern. Med. 153, 182-193. doi: 10.7326/00034819-153-3-201008030-00258

Reinvang, I., Deary, I. J., Fjell, A. M., Steen, V. M., Espeseth, T., and Parasuraman, R. (2010). Neurogenetic effects on cognition in aging brains: a window of opportunity for intervention? Front. Aging Neurosci. 2:143. doi: 10.3389/fnagi.2010.00143

Reinvang, I., Espeseth, T., and Westlye, L. T. (2013). APOE-related biomarker profiles in non-pathological aging and early phases of Alzheimer's disease. Neurosci. Biobehav. Rev. 37, 1322-1335. doi: 10.1016/j.neubiorev.2013. 05.006

Risacher, S. L., Kim, S., Shen, L., Nho, K., Faroud, T., Green, R. C., et al. (2013). The role of apolipoprotein E (APOE) genotype in early mild cognitive impairment (E-MCI). Front. Aging Neurosci. 5:11. doi: 10.3389/fnagi.2013.00011

Ritchie, K., Artero, S., and Touchon, J. (2001). Classification criteria for mild cognitive impairment: a population-based validation study. Neurology 56, 37-42. doi: 10.1212/WNL.56.1.37

Rowe, J. B., Hughes, L., Williams-Gray, C. H., Bishop, S., Fallon, S., Barker, R. A., et al. (2010). The val158met COMT polymorphism's effect on atrophy in healthy aging and Parkinson's disease. Neurobiol. Aging 31, 1064-1068. doi: 10.1016/j.neurobiolaging.2008.07.009

Sapkota, S., Vergote, D., Westaway, D., Jhamandas, J., and Dixon, R. A. (2014). Synergistic associations of catechol-O-methyltransferase and brain-derived neurotrophic factor with executive function in aging are selective and modified by apolipoprotein E. Neurobiol. Aging. doi: 10.1016/j.neurobiolaging.2014.06.020. [Epub ahead of print].

Savitz, J., Solms, M., and Ramesar, R. (2006). The molecular genetics of cognition: dopamine, COMT and BDNF. Genes Brain Behav. 5, 311-328. doi: 10.1111/j.1601-183X.2005.00163.x

Schiepers, O. J. G., Harris, S. E., Gow, A. J., Pattie, A., Brett, C. E., Starr, J. M., et al. (2012). APOE E4 status predicts age-related cognitive decline in the ninth decade: longitudinal follow-up of the Lothian Birth Cohort 1921. Mol. Psychiatry 17, 315-324. doi: 10.1038/mp.2010.137

Small, B. J., Rosnick, C. B., Fratiglioni, L., and Bäckman, L. (2004). Apolipoprotein E and cognitive performance: a meta-analysis. Psychol. Aging 19, 592-600. doi: 10.1037/0882-7974.19.4.592

Sperling, R. A., Karlawish, J., and Johnson, K. A. (2013). Preclinical Alzheimer's disease: the challenges ahead. Nat. Rev. Neurol. 9, 54-58. doi: 10.1038/nrneurol.2012.241

Tapia-Arancibia, L., Aliaga, E., Silhol, M., and Arancibia, S. (2008). New insights into brain BDNF function in normal aging and Alzheimer disease. Brain Res. Rev. 59, 201-220. doi: 10.1016/j.brainresrev.2008.07.007

Thurstone, T. G. (1962). Primary Mental Abilities: Grades 9-12, 1962 Revision. Chicago, IL: Science Research Associates.

Vandermorris, S., Hultsch, D. F., Hunter, M. A., MacDonald, S. W. S., and Strauss, E. (2011). Including persistency of impairment in mild cognitive impairment classification enhances prediction of 5-year decline. Arch. Clin. Neuropsychol. 26, 26-37. doi: 10.1093/arclin/acq093

Wechsler, D. (1981). WAIS-R Manual. New York, NY: Psychological Corporation. Winblad, B., Palmer, K., Kivipelto, M., Jelic, V., Fratiglioni, L., Wahlund, L.-O., et al. (2004). Mild cognitive impairment—beyond controversies, towards a consensus. Report of the International Working Group on Mild Cognitive Impairment. J. Int. Med. 256, 240-246. doi: 10.1111/j.1365-2796.2004. 01380.x

Wisdom, N. M., Callahan, J. L., and Hawkins, K. A. (2011). The effects of apolipoprotein $\mathrm{E}$ on non-impaired cognitive functioning: a 
meta-analysis. Neurobiol. Aging 32, 63-74. doi: 10.1016/j.neurobiolaging.2009. 02.003

Wishart, H. A., Roth, R. M., Saykin, A. J., Rhodes, C. H., Tsongalis, G. J., Pattin, K. A., et al. (2011). COMT Val158Met genotype and individual differences in executive function in healthy adults. J. Int. Neuropsychol. Soc. 17, 174-180. doi: $10.1017 /$ S1355617710001402

Woodard, J. L., Sugarman, M. A., Nielson, K. A., Smith, J. C., Seidenberg, M., Durgerian, S., et al. (2012). Lifestyle and genetic contributions to cognitive decline and hippocampal structure and function in healthy aging. Curr. Alzheimer Res. 9, 436-446. doi: 10.2174/156720512800492477

Yarnell, A. J., Breen, D. P., Duncan, D. P., Khoo, T. K., Coleman, S. Y., Firbank, M. J., et al. (2014). Characterizing mild cognitive impairment in incident Parkinson disease: The ICICLE-PD Study. Neurology 82, 308-316. doi: 10.1212/WNL.0000000000000066

Zheng, J., Dixon, R. A., and Li, L. (2012). Development of isotope labelling LC-MS for human salivary metabolomics and application to profiling metabolome changes associated with mild cognitive impairment. Anal. Chem. 84, 10802-10811. doi: 10.1021/ac3028307
Conflict of Interest Statement: The authors declare that the research was conducted in the absence of any commercial or financial relationships that could be construed as a potential conflict of interest.

Received: 02 January 2014; accepted: 19 August 2014; published online: 05 September 2014.

Citation: Dixon RA, DeCarlo CA, MacDonald SWS, Vergote D, Jhamandas J and Westaway D (2014) APOE and COMT polymorphisms are complementary biomarkers of status, stability, and transitions in normal aging and early mild cognitive impairment. Front. Aging Neurosci. 6:236. doi: 10.3389/fnagi.2014.00236

This article was submitted to the journal Frontiers in Aging Neuroscience.

Copyright $\odot 2014$ Dixon, DeCarlo, MacDonald, Vergote, Jhamandas and Westaway. This is an open-access article distributed under the terms of the Creative Commons Attribution License (CC BY). The use, distribution or reproduction in other forums is permitted, provided the original author (s) or licensor are credited and that the original publication in this journal is cited, in accordance with accepted academic practice. No use, distribution or reproduction is permitted which does not comply with these terms. 